\title{
Animal magnetocardiography using superconducting quantum interference device gradiometers assisted with magnetic nanoparticle injection: A sensitive method for early detecting electromagnetic changes induced by hypercholesterolemia
}

\author{
C. C. Wu \\ Department of Primary Care Medicine, College of Medicine, National Taiwan University, Taipei 106, \\ Taiwan; Department of Internal Medicine, College of Medicine, National Taiwan University, Taipei 106, \\ Taiwan; and Department of Internal Medicine, E-Da Hospital, Kaohsiung 824, Taiwan
}

B. F. Hong, B. H. Wu, S. Y. Yang, and H. E. Horng ${ }^{\text {a),b),c) }}$

Institute of Electro-optical Science and Technology, National Taiwan Normal University, Taipei 116, Taiwan

H. C. Yang ${ }^{\text {b),d) }}$

Department of Physics, National Taiwan University, Taipei 106, Taiwan

W. Y. Isaac Tseng

Center for Optoelectronic Biomedicine, National Taiwan University Hospital, Taipei 106, Taiwan

W. K. Tseng, Y. B. Liu, L. C. Lin, and L. S. Lu

Department of Internal Medicine, College of Medicine, National Taiwan University, Taipei 106, Taiwan and Department of Internal Medicine, E-Da Hospital, Kaohsiung 824, Taiwan

\author{
Y. H. Lee \\ Bio-signal Research Center, Korea Research Institute of Standards and Science, Daejeon 305-340, \\ Republic of Korea
}

(Received 4 November 2006; accepted 18 December 2006; published online 2 February 2007)

In this work, the authors used a superconducting quantum interference device (SQUID) magnetocardiography (MCG) system consisted of 64-channel low-transition-temperature SQUID gradiometers to detect the MCG signals of hepercholesterolemic rabbits. In addition, the MCG signals were recorded before and after the injection of magnetic nanoparticles into the rabbits' ear veins to investigate the effects of magnetic nanoparticles on the MCG signals. These MCG data were compared to those of normal rabbits to reveal the feasibility for early detection of the electromagnetic changes induced by hypercholesterolemia using MCG with the assistance of magnetic nanoparticle injection. (c) 2007 American Institute of Physics. [DOI: 10.1063/1.2433036]

Due to its ultrahigh sensitivity in magnetic flux, superconducting quantum interference devices (SQUIDs) are widely applied to detect small magnetic signals. ${ }^{1-3}$ One of the important applications is to detect the spatial and temporal biomagnetic signals of the heart, so-called magnetocardiography (MCG), ${ }^{4-7}$ which are generated by the electric activities of beating hearts. Several groups have reported the relationships between the MCG and the physiology of heart. ${ }^{4-6}$ Furthermore, the diagnostic models on animals having heart diseases using SQUID MCG systems have been established. ${ }^{7}$ These published papers show advantages such as high sensitivity, high accuracy, noncontact, noninvasive, and high convenience for diagnosing heart diseases using SQUID MCG data. Hence, the developments of SQUID MCG in aspects of technologies and clinical trials have been attracting lots of interests of engineers, scientists, and even medical doctors.

On the other hand, nanoparticles are very useful in achieving bioimage. For example, biofunctionalized semiconductor quantum dots are used as markers for imaging

\footnotetext{
${ }^{a)}$ Also at: Department of Physics, National Taiwan Normal University, Taipei 116, Taiwan.

b) Author to whom correspondence should be addressed.

${ }^{c}$ Electronic mail: phyfv001@scc.ntnu.edu.tw

${ }^{d)}$ Electronic mail: hcyang@phys.ntu.edu.tw
}

specific organs, lymph nodes, and of other beings in vivo. ${ }^{8,9}$ Contrast to optical bioimages using semiconductor quantum dots, magnetic nanoparticles may contribute to magneticrelated biosignals via utilizing its magnetism. In addition, with the superparamagnetism, magnetic nanoparticles may be applied not only to static but also to dynamic magnetobioimaging. These motivated us to investigate the effects of magnetic nanoparticles on the MCG signals, especially of the disease models. To do this, a low-transition-temperature (low- $T_{c}$ ) SQUID MCG system was used to detect the MCG signals of rabbits injected intravenously with magnetic nanoparticles. The MCG signals before and after nanoparticle injection were compared to clarify the effects of magnetic nanoparticles on the MCG signals. The MCG data from normal and hepercholesterolemic rabbits were compared to test the feasibility for early detection of the electromagnetic changes induced by hypercholesterolemia using MCG with the assistance of magnetic nanoparticle injection.

The SQUID MCG system consisted of 64 low- $T_{c}$ SQUID gradiometers, which was developed by Korea Research Institute of Standards and Science. The MCG system was set up inside a magnetically shielded room (MSR). Inside the MSR, the SQUID gradiometers exhibit a noise level of about $50 \mathrm{fT} / \mathrm{Hz}^{1 / 2}$ at $1 \mathrm{~Hz}$. 
(a)

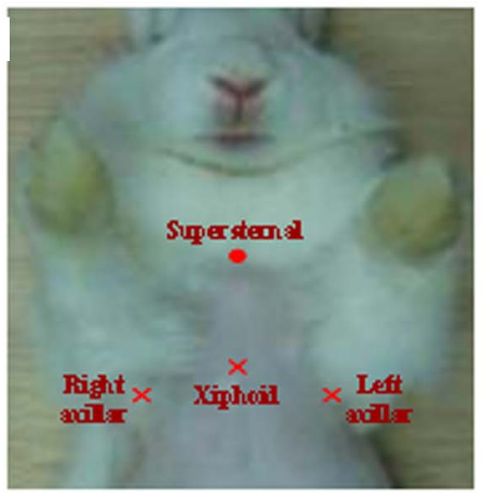

(b)
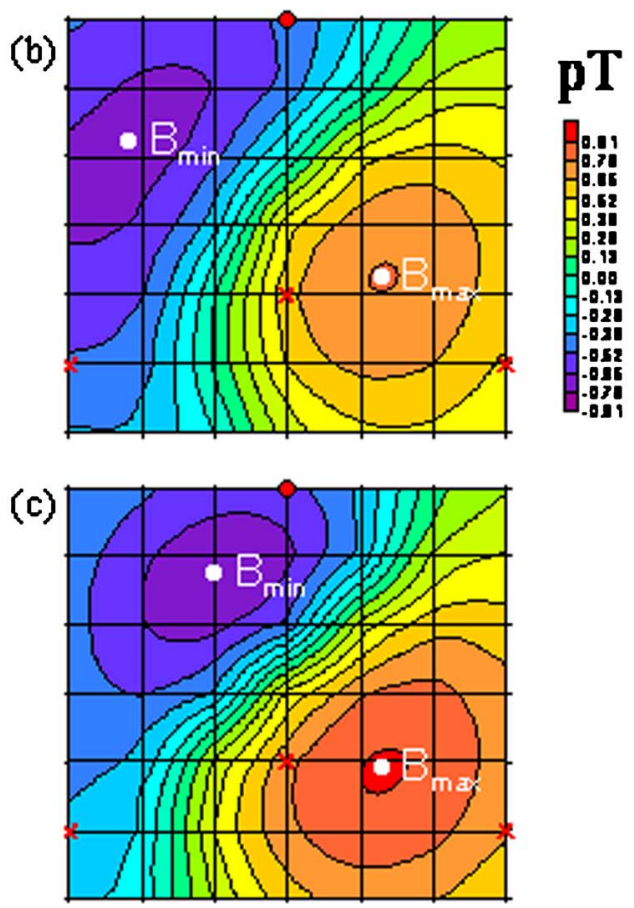

FIG. 1. (Color online) (a) Picture to show the chest area, which is enveloped with supersternal, right axillar, and left axillar, for MCG measurement. Typical MCG contour map for $Q$ wave of a hepercholesterolemic rabbit (b) before and (c) after being injected with magnetic fluid.

Three normal and another three hypercholesterolemic rabbits were used for MCG studies. To make the rabbits hypercholesterolemic, we fed the rabbits with standard rabbit chow enriched with $0.5 \%$ cholesterol, $0.2 \%$ vitamins, $0.8 \%$ minerals, and $12.1 \%$ coconut oil for 3 weeks. Statistically, the serum concentrations of cholesterol were around 500 and $50 \mathrm{mg} / \mathrm{dL}$ for hepercholesterolemic and normal rabbits, respectively. For MCG measurement, each rabbit was anesthetized. The MCG measurement was performed just before the injection of magnetic nanoparticles. The following MCG measurement was done immediately after the injection. The following MCG measurements were conducted every $20 \mathrm{~min}$. The other normal rabbit was used for magnetic resonance imaging (MRI) study before and after the injection of magnetic nanoparticles. Experiments on rabbits were performed in accordance with the regulations set by the National Taiwan University Animal Care and Use Committee and approved by National Taiwan University.

The injected magnetic nanoparticles are dextran-coated $\mathrm{Fe}_{3} \mathrm{O}_{4}$ nanoparticles (GABC Co.), which are homogeneously dispersed in water. The mean diameter of the nanoparticles is $52 \mathrm{~nm}$. The saturated magnetization of the magnetic fluid Downloaded 24 Jul 2009 to 140.112 .113 .225 . Redistribution subject

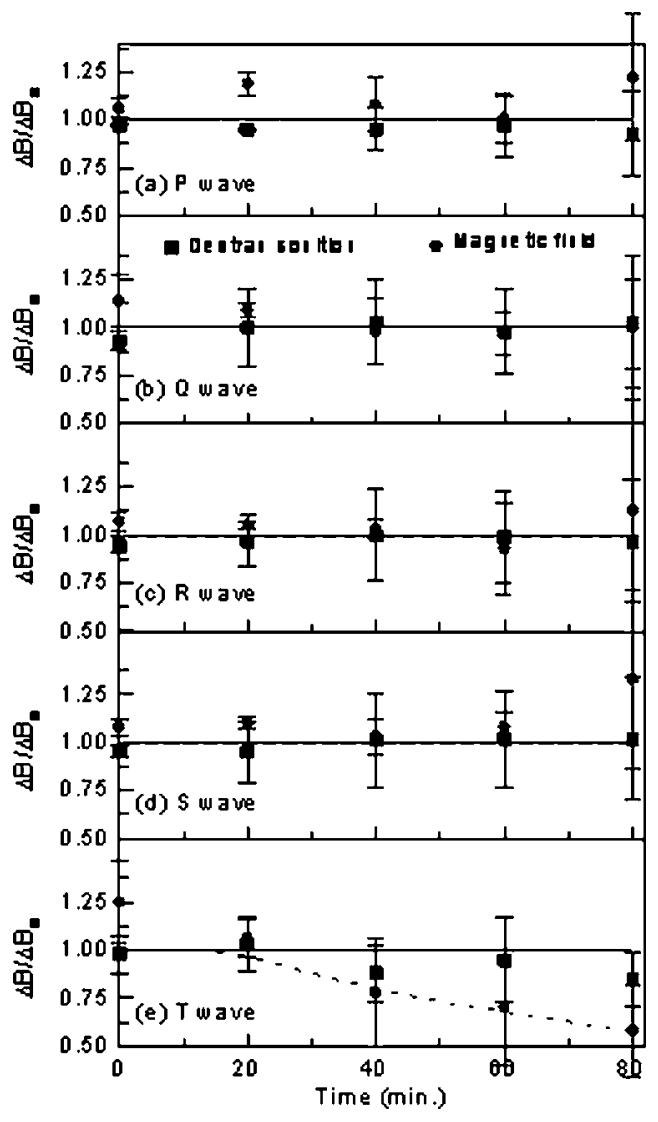

FIG. 2. Temporal variations in the normalized peak-to-peak magnetic signal, $\Delta B / \Delta B_{0}$, in the MCG contour map for (a) $P$, (b) $Q$, (c) $R$, (d) $S$, and (e) $T$ waves of normal rabbits injected with magnetic fluid (dashed lines) and pure dextran solution (solid lines).

containing magnetic nanoparticles is $0.3 \mathrm{emu} / \mathrm{g}$. The volume of magnetic fluid injected into each rabbit was $2 \mathrm{ml}$. The magnetic fluid was injected into the rabbits' ear vein. Hemodynamically, the injected magnetic fluid flew from the right to the left heart, and then perfused the myocardium through the coronary arteries. It was observed that the magnetic fluid flew into the heart in a short period of time after injection, as evidenced by the MRI study (not shown here).

The MCG signals within the chest area of a hypercholesterolemic rabbit were detected, as shown in Fig. 1(a). The typical averaged MCG contour map within the detected area for a certain instant, say, $Q$ wave, is plotted in Fig. 1(b). The maximum intensity $B_{\max }$ was observed at the lower-right part, and the minimum intensity $B_{\min }$ occurred at the upperleft part. After the injection of magnetic fluid, the MCG contour map of $Q$ wave is probed, as shown in Fig. 1(c). It was found that the $B_{\max }$ became higher than that before the injection. To quantitatively describe the variation in the intensities of MCG contour maps due to the injection of magnetic fluid, the peak-to-peak magnetic signal $\Delta B$, defined as $B_{\max }-B_{\min }$, was analyzed as a function of time after the injection of magnetic fluid into rabbits. To eliminate the individual difference among rabbits, the $\Delta B$ was normalized to $\Delta B_{0}$, which corresponds to $B_{\max }-B_{\min }$ just before the injection of magnetic fluid. Since the magnetic nanoparticles $\mathrm{Fe}_{3} \mathrm{O}_{4}$ are coated with dextran, the effect on $\Delta B$ may be attributed to the $\mathrm{Fe}_{3} \mathrm{O}_{4}$ magnetic nanoparticles or dextran. To clarify this point, the time-dependent $\Delta B / \Delta B_{0}$ for the rabbits injected with pure dextran solution was also investigated. The timedependent normalized values, $\Delta B / \Delta B_{0}$, for $P, Q, R, S$, and $T$
to AlP license or copyright; see http://apl.aip.org/apl/copyright.jsp 


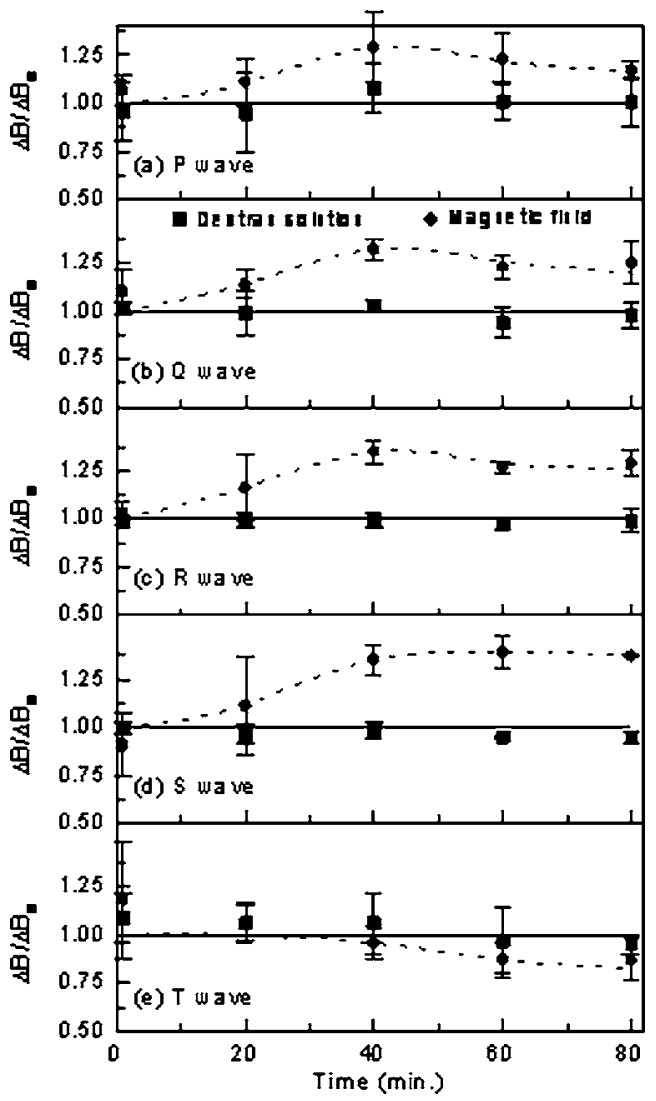

FIG. 3. Temporal variations in the normalized peak-to-peak magnetic signal, $\Delta B / \Delta B_{0}$, in the MCG contour map for (a) $P$, (b) $Q$, (c) $R$, (d) $S$, and (e) $T$ waves of hypercholesterolemic rabbits injected with magnetic fluid (dashed lines) and pure dextran solution (solid lines).

waves for normal and hypercholesterolemic rabbits are plotted in Figs. 2 and 3, respectively. The data at time being zero correspond to $\Delta B / \Delta B_{0}$ 's before the injection of magnetic fluid. The squares and solid lines represent for the data after dextran injection, and the circles and dashed lines for those after magnetic fluid injection, respectively.

In order to identify any effect on $\Delta B$ of the $P, Q, R$, and $S$ waves, the MCG contour map of normal rabbits injected with magnetic fluid or pure dextran solution were measured. Furthermore, the $\Delta B / \Delta B_{0}$ as a function of time was analyzed and is shown in Fig. 2. It is obvious that both dashed lines and solid lines in Figs. 2(a)-2(d) for $P, Q, R$, and $S$ waves overlap and are kept at a constant value of 1 . This means that both magnetic fluid and pure dextran solution hardly affect the cardiac electromagnetic activities during $P$ to $S$ wave. However, the data shown in Fig. 2(c) reveal that the $\Delta B$ in the MCG contour map for $T$ wave was depressed when normal rabbits were injected with magnetic fluid. The suppressed $\Delta B$ was probable due to most of the magnetic nanoparticles flowing downstream through the coronary arteries during the cardiac diastolic phase, which was compatible with $T$ wave on MCG tracings.

In contrast, it could be observed in Fig. 3 that, except that of $T$ wave, the time-dependent $\Delta B / \Delta B_{0}$ curves for $P, Q$,
$R$, and $S$ waves exhibited a similar behavior. The $\Delta B / \Delta B_{0}$ 's became higher than 1 after the injection of magnetic fluid. This result implies that the difference between $B_{\max }$ and $B_{\min }$ in the MCG contour map was enhanced for hypercholesterolemic rabbits after the injection of magnetic fluids. The results are shown with solid lines in Figs. 3(a)-3(e) for $P, Q$, $R, S$, and $T$ waves, respectively. Remarkably, the $\Delta B / \Delta B_{0}$ almost remains at 1 for the solid lines in Figs. 3(a)-3(e). This reveals that the enhancement in $\Delta B$ in the MCG contour map for $P, Q, R$, and $S$ waves of hypercholesterolemic rabbits was caused only by the $\mathrm{Fe}_{3} \mathrm{O}_{4}$ magnetic nanoparticles. Although the detailed mechanism responsible for the enhancement in $\Delta B$ of MCG contour map is not clear, it is strongly suggested that the $\mathrm{Fe}_{3} \mathrm{O}_{4}$ magnetic nanoparticles leaking from the coronary arteries into myocardium through tiny clefts of the coronary arteries of hypercholesterolemic rabbits may be the most possible candidate. Thus, the electromagnetic signals generated by the action potential of myocardium are modified with the surrounding magnetic nanoparticles.

Through comparing the magnetic-fluid induced variations in $\Delta B$ of MCG contour maps in Figs. 2 and 3, a significantly different behavior between normal and hypercholesterolemic rabbits was obtained. It is worthy to note that the conventional diagnosis such as electrocardiography cannot distinguish the difference between normal and hypercholesterolemic rabbits until having been hypercholesterolemic for 6 weeks. ${ }^{10}$ However, according to the results shown in Figs. 2 and 3, an abnormal magnetic-fluid induced $\Delta B$ of MCG contour map could be observed in rabbits having hypercholesterolemia only for 3 weeks. Therefore, the measurement of magnetic-fluid induced $\Delta B$ in MCG is promising for the early-stage diagnosis for hypercholesterolemic rabbits.

This work is supported by the National Science Council of Taiwan under Grant Nos. 95-2120-M-003-001, 95-2112M-003-017-MY2, 95-2112-M-003-019, 95-2221-E-212-054MY3, and 95-2752-M-002-016-PAE.

${ }^{1}$ C. Carr, A. Cochran, J. Kuznik, D. McA. McKirdy, and G. B. Donaldson, Cryogenics 36, 691 (1996).

${ }^{2}$ A. H. Miklich, D. Koelle, F. Ludwig, D. T. Nemeth, E. Dantsker, and J. Clarke, Appl. Phys. Lett. 66, 230 (1995).

${ }^{3}$ H. E. Horng, S. Y. Yang, Chin-Yih Hong, C. M. Liu, P. S. Tsai, H. C. Yang, and C. C. Wu, Appl. Phys. Lett. 88, 252506 (2006).

${ }^{4}$ Y. Tavrin, Y. Zhang, M. Mück, A. I. Brazinski, and C. Heiden, Appl. Phys. Lett. 62, 1824 (1993).

${ }^{5}$ K. Yokosawa, A. Tsukamoto, A. Suzuki, T. Miyashita, M. Ogata, Y. Seki, and K. Tsukada, Supercond. Sci. Technol. 16, 1383 (2003).

${ }^{6}$ K. Kim, H. Kwon, Y. H. Lee, J. M. Kim, Y. K. Park Y. G. Ko and N. Chung, J. Korean Phys. Soc. 48, 1123 (2006).

${ }^{7}$ H. C. Yang, T. Y. Wu, H. E. Horng, C. C. Wu, S. Y. Yang, S. H. Liao, C. H. Wu, J. T. Jeng, J. C. Chen, K. L. Chen, and M. J. Chen, Supercond. Sci. Technol. 19, S297 (2006).

${ }^{8}$ Y. M. Huh and Y. J. Kim, Curr. Appl. Phys. 6, e22 (2006).

${ }^{9}$ F. Pinaud, X. Michalet, L. A. Bentolila, J. M. Tsay, S. Doose, J. J. Li, G. Iyer, and S. Weiss, Biomaterials 27, 1679 (2006).

${ }^{10}$ Y. B. Liu, C. C. Wu, L. S. Lu, M. J. Su, C. W. Lin, S. F. Lin, L. S. Chen, M. Fishbein, and Y. T. Lee, Circ. Res. 30, 1145 (2003). 